\title{
Establishing Benchmarks for Helicopter EMS Patient Stabilization Times in Interfacility Transport for Primary Percutaneous Coronary Intervention*
}

\author{
Loren Brown $^{1,2}$, Annette Arthur ${ }^{1,2}$, Claudia Keeling ${ }^{1,2}$, Christine Yuhas ${ }^{1,2}$, Stephen H. Thomas ${ }^{1,2}$ \\ ${ }^{1}$ Air Evac Lifeteam Oklahoma, West Plains, USA; ${ }^{2}$ School of Community Medicine, Department of Emergency Medicine, Univer- \\ sity of Oklahoma Tulsa, USA. \\ Email: Stephen-Thomas@OUHSC.edu
}

Received October $24^{\text {th }}, 2012$; revised November $27^{\text {th }}, 2012$; accepted December $11^{\text {th }}, 2012$

\begin{abstract}
Helicopter EMS (HEMS) allows for patients to be quickly transported into regional cardiac centers, often to receive primary percutaneous coronary intervention (PCI). Since PCI is a time-critical therapy, it is important that patients get to primary PCI as quickly as possible. HEMS crews' "on-scene” times for trauma patients have been extensively studied, and recent years have seen many efforts to minimize the time required to prepare patients for transport. There has been less attention to interfacility transport "scene times" for HEMS crews at referring hospitals; this includes stabilization times for preparing cardiac patients for loading onto aircraft for HEMS transport to primary PCI. In the absence of guiding evidence, system benchmarking and quality improvement are difficult. Therefore the current study was undertaken, to assess and describe the HEMS crew "on-scene" times or "patient stabilization times" (PSTs) at referring hospitals, for interfacility transported cardiac patients flown for primary PCI. Descriptive analysis identified a PST median of 19 minutes (interquartile range 15 - 24), and univariate analyses using Kruskal-Wallis testing found no association between prolonged PST and sending unit type (Emergency Department versus other), off-hours transports, or relatively frequent (at least monthly) use of HEMS ( $p$ for all comparisons $>0.64$ ). Outlier PSTs, defined a priori as those exceeding the median by at least a half-hour, were found in $12 \%$ of all cases. These data could be useful as a starting point for system planning and benchmarking efforts in regionalized systems of acute cardiac care.
\end{abstract}

Keywords: Helicopter EMS; Percutaneous Coronary Intervention; Regional Cardiac Care; Benchmarking

\section{Introduction}

"On-scene" time for helicopter EMS (HEMS) crews has traditionally been applied to time spent in crews' pretransport patient stabilization at out-of-hospital trauma scenes. With more scrutiny on HEMS benefits for interfacility transports, assessment for prolonged referring hospital "on-scene" or "patient stabilization" times (hereafter, PSTs) warrants attention since prolonged PST can reduce or even negate benefit of HEMS transport [1]. Efficient movement is particularly critical in the patient population undergoing HEMS transfers directly to cardiac catheterization laboratories for emergency percutaneous coronary intervention (PCI). For this tine-critical therapy, defined herein as the deployment of the first device (e.g. balloon inflation), HEMS transport goals include (but are not limited to) getting patients to PCI within the least amount of time possible [2]. Not only are PCI patients needing to reach PCI centers within certain time windows,

*HEMS patient stabilization times for PCI transports. there is also clear evidence supporting desirability of savings of time within the window (e.g. 50 minutes is better than 80 minutes) [3].

Despite the importance of moving as quickly as patient and transport safety will allow, there are few data providing benchmarks for HEMS crew PST at referring hospitals. Systems' efforts at measuring and improving time performance starts with comparing current performance to broader standards; the goal of this study was to assess and describe PST times in one system, to provide benchmarking information. Secondary study goals included assessment as to whether there were associations between PST and the following independent variables: Emergency Department (ED) as compared to non-ED sending unit; daytime versus off-hours (1900-0700) transports; or frequent (more than twice-monthly) HEMS use for PCI. Finally, the study set out to define the frequency of occurrence of "outlier" times (defined as PSTs of more than halfhour). 


\section{Methods}

\subsection{Design}

This was a retrospective review of a consecutive series of interfacility transports for emergency PCI (i.e. directly to cardiac catheterization suites), occurring during the first quarter of 2012.

\subsection{Setting}

The study was conducted in a rural southwestern US state (Oklahoma). The study HEMS program comprises 8 helicopters, performing roughly 3000 flights annually, using a nurse/paramedic configuration. Institutional Review Board for the study was obtained from the University of Oklahoma.

\subsection{Subjects and Data Collection}

Data were collected from flight records of all patients undergoing interfacility transport for primary PCI, by the study service, over the first quarter of 2012. Flight crews routinely and prospectively record the times of arrival at patients' bedsides and also the time of departure from the patients' bedside to head to the aircraft for the flight to PCI. The time interval between HEMS crew arrival at patients' bedside, to departure with the "packaged" patient from the sending facility unit to go to the aircraft, defined the study's primary endpoint of "patient stabilization time" or PST. Additional data assessed for the study included time of transport initiation (i.e. initial HEMS dispatch), identity of sending facility (i.e. hospital name) and unit (ED versus other).

\subsection{Analysis}

Descriptive calculations focused on measures of central tendency of PST: medians with interquartile range (IQR) and means with standard deviation (SD). Additional descriptive reporting including the frequency of transports for which the PST was at least half-hour longer than the median (these times were defined as "outlier" times). Univariate analysis with Kruskal-Wallis testing was performed to assess for association between PST time and whether transports were "off-hours" (between 1900-0700), whether transporting units were ED or non-ED, and whether transporting hospitals were frequent (at least twice-monthly) or infrequent utilizers of HEMS for PCI transports. Hypothesis-generating analysis was also planned to assess for association between PST and male/female status or older (>65 years) versus younger age. Statistical analysis was performed with Stata 12 MP (StataCorp, College Station TX) and significance was defined at the $p<0.05$ level.

\section{Results}

Overall, there were 42 transports comprising the study dataset. Transports came from 7 HEMS bases, with one base accounting for 19 (45\%) of cases and the remaining bases with 7 or fewer cases. Patients ranged in age from 40 to 82 , and 33 (79\%) were male. One referring hospital accounted for 12 (29\%) of the total study population, with the remaining 15 hospitals accounting for 7 or fewer cases.

\subsection{Median and Mean Patient Stabilization Times}

For the overall dataset of 42 cases, the HEMS PST was a median 18.5 minutes with IQR 15 - 24. The 95\% CI for the mean of 19.9 minutes was 17.4 to 22.5 minutes. In 7 cases (16.7\%) the PST was 10 minutes or less. In 5 cases (11.9\%) PST exceeded a half-hour.

\subsection{Univariate Analyses}

There were no significant associations identified between independent variables analyzed, and the dependent variable of PST. PST was no different for off-hours as compared to daytime transports $(p=0.92)$, frequent (at least once-monthly) HEMS use for PCI as compared to less frequent use $(p=0.64)$, or ED versus non-ED sending unit $(p=0.92)$. There was also no association between PST and male/female status $(p=0.41)$ or older age $(>65$ years) versus younger adults $(p=0.23)$.

\section{Limitations}

The current study has a number of limitations. These shortcomings are important to acknowledge, and they should preclude extension of these results beyond the intended goal of providing a general benchmark for PST.

\subsection{Patient Clinical Characteristics}

First and most importantly, the results are not adjusted for important patient clinical characteristics. These characteristics are not available from the database from which the study was performed. Such characteristics include parameters such as intubation status and need for critical resuscitation at referring hospitals during the "PST" interval. These types of cases, in which PST might be necessarily prolonged, are expected to be covered within the PST ranges and findings reported herein (e.g. the $12 \%$ of cases in which PST exceeded a half-hour). In short, the current study did not address the "why" of PST results in any given instance. Further study should include detailed clinical parameters and identify the reasons for PST prolongation, in an attempt to assess whether those delays are amenable to reduction with education directed at referring 
hospitals.

\subsection{Study $n$}

A second limitation is related to the number of cases comprising the study population. The case series size was sufficient for the main goal of describing PST intervals in our setting (as evidenced by the relatively narrow IQR and CI results). However, the failure to identify significant predictors of prolonged PST among the tested independent variables could be related to insufficient power. Follow-up research should include a sufficiently large population to enable both rigorous univariate analyses, and also adjusted analyses allowing rigorous search for remediable factors contributing to prolonged PST.

\subsection{Retrospective Design}

Like any retrospective study, the current analysis risks imprecision due to the fact that study data were assessed months after the information was collected. Because of the importance of time information to prehospital care, and due to the fact that the study variables are (and have been) routinely collected and recorded by HEMS crews, it is not likely that errors in data collection or recording impacted the study results in significant fashion.

\section{Discussion and Conclusions}

The current data are intended for use as a general guideline for cardiac care systems considering evaluation and quality improvement of PSTs. The results of this study provide approximations of the range of results that may be expected in similar systems of care. For across-theboard measurements, 20 minutes appears to be a reasonable goal for PST; it should be expected that about $12 \%$ of the time, PST may be significantly longer. On the other end of the PST spectrum, systems should expect to be able to frequently (nearly $17 \%$ in this series) achieve PSTs of less than 10 minutes.

The upper range of the IQR for PST in this study, of 24 minutes, compares relatively favorably with the median (18.5 minutes) but does suggest potential for some room for improvement. In a diagnostic category (i.e. STelevation myocardial infarction) in which savings of even a few minutes has been consistently identified as important [4], the "spread" of PSTs as indicated by the IQR could also be useful as an indicator of system function; the narrower the IQR the less "variability" in the system.

System benchmarking is an important part of optimizing care in regionalized systems of care for acute cardiac patients. Previous studies have emphasized the utility of studying various components of the timeframe from initial onset of symptoms to the final step of performance of device deployment during PCI [5]. While this study fo- cused on just one part of the process, it appears quite possible that with proper education of referring facility providers, the PST interval can be significantly reduced. Among the important steps that may be saved with respect to patient preparation for HEMS transport are early completion and assembly of paperwork, discontinuation of unnecessary infusions, and management of airways as dictated by clinical circumstances. Of course, one of the steps entailed in optimizing PST is collaboration between referring facilities, transporting services, and receiving facilities. Fortunately, the literature demonstrates that this type of collaboration is both feasible and effective in producing optimally performance in regionalized cardiac care systems [4,5].

These PST results in PCI patients should be applied to other patient populations only with caution. For this study set, the patients that were analyzed were a very clearly defined group of those who were being emergently transported for primary PCI. Other populations - even other cardiac populations-are sufficiently different in terms of acuity, management, or time-sensitivity, that the results of this study are best used only as a general guide.

\section{Conclusion}

In conclusion, the current study results suggest that systems of cardiac care can compare their PST benchmarks to approximately 20 minutes on average, with about $10 \%$ of cases requiring half-hour or more longer and about $17 \%$ of cases executable within 10 minutes. These numbers are proposed not as rigid benchmarks, but rather as general guides for comparative purposes as systems try and maximize efficiency.

\section{REFERENCES}

[1] S. H. Thomas and A. O. Arthur "Helicopter EMS: Research Endpoints and Potential Benefits,” Emergency Medicine International, Vol. 2012, 2012, Article ID 698562.

[2] D. S. Pinto, A. J. Kirtane, B. K. Nallamothu, et al., "Hospital Delays in Reperfusion for ST-Elevation Myocardial Infarction: Implications When Selecting a Reperfusion Strategy," Circulation, Vol. 114, No. 19, 2006, pp. 20192025. doi:10.1161/CIRCULATIONAHA.106.638353

[3] B. K. Nallamothu, E. H. Bradley and H. M. Krumholz, "Time to Treatment in Primary Percutaneous Coronary Intervention,” New England Journal of Medicine, Vol. 357, No. 16, 2007, pp. 1631-1638. doi:10.1056/NEJMra065985

[4] S. H. Thomas, C. Kociszewski, R. J. Hyde, P. J. Brennan and S. K. Wedel, "Prehospital Electrocardiogram and Early Helicopter Dispatch to Expedite Interfacility Transfer for Percutaneous Coronary Intervention,” Critical Pathways in Cardiology, Vol. 5, No. 3, 2006, pp. 155-159. doi:10.1097/01.hpc.0000234809.93495.e3

[5] J. C. Blankenship, T. A. Haldis, G. C. Wood, K. A. Skeld- 
ing, T. Scott and F. J. Menapace, "Rapid Triage and Transport of Patients with ST-Elevation Myocardial Infarction for Percutaneous Coronary Intervention in a Rural Health
System,” American Journal of Cardiology, Vol. 100, No. 6, 2007, pp. 944-948. doi:10.1016/j.amjcard.2007.04.031 